\title{
PATIENTS' EXPECTATIONS AND POST-BARIATRIC SURGERY SATISFACTION
}

Garrido, Susana; Silva, I; Gonçalves, I; Caldas, AR; Cardoso, MH

\section{INTRODUCTION}

Treatment satisfaction is simultaneously a cause and consequence of the success of bariatric surgery - not only it is a reflection of the efficacy of treatment, it also enhances weight loss. A questionnaire that evaluates this variable has recently been created and validated by the authors (PostBariatric Surgery Satisfaction Questionnaire).

\section{AIMS}

To identify patients' expectations regarding bariatric surgery; to identify the domains that contribute to the degree of satisfaction after bariatric surgery

\section{METHODS}

- Patients submitted to bariatric surgery, presenting to our Obesity Outpatient Clinic between June-December/2013

- Patients' Expectations Questionnaire (improve your self-esteem, improve the way other people see you, improve your health status, improve your sex life, be able to care for personal hygiene independently, be able to move better, be able to wear clothes you like, improve your professional status, improve your love life, lose weight, etc.)

- Post-Bariatric Surgery Satisfaction Questionnaire

(22 items, 5-point scale $-1=$ very dissatisfied, $5=$ very satisfied)

\section{RESULTS}

\section{CHARACTERISTICS OF THE STUDIED POPULATION $(n=86)$}

Gender (male/female), \%

$14 / 86$

Age, years

$50(19 ; 23-73)$

Type of surgery (laparoscopic gastric

bypass/adjustable gastric banding), $\%$

Initial BMI, $\mathrm{kg} / \mathrm{m}^{2}$ a

$67 / 33$

$45.9(8.4 ; 32.1-84.4)$

Follow up time since surgery, months a

$37(56 ; 1-220)$

\section{EXPECTATIONS REGARDING TREATMENT}

\begin{tabular}{|c|c|c|c|c|}
\hline $\begin{array}{c}\text { Actual BMI } \\
\left(\mathrm{kg} / \mathrm{m}^{2}\right)^{\mathrm{a}}\end{array}$ & $\begin{array}{c}31.7 \\
(9.6 ; 23.5-51.2)\end{array}$ & VS & $\frac{\text { Idealized BMI }}{\left(\mathrm{kg} / \mathrm{m}^{2}\right)^{a}}$ & $\begin{array}{c}\mathbf{2 7 . 7} \\
(5.5 ; 22.9-41.6)\end{array}$ \\
\hline $\begin{array}{c}\text { Actual \% excess } \\
\text { BMI lost }{ }^{\text {a }}\end{array}$ & $\begin{array}{c}68.8 \\
(34.6 ; 8.2-113.3)\end{array}$ & VS & $\frac{\text { Idealized } \% \text { excess }}{\text { BMI lost }}$ & $\begin{array}{c}85.1 \\
(20.7 ; 33.2-112.6)\end{array}$ \\
\hline
\end{tabular}

${ }^{a}$ Data expressed as median (IQR, min-max)

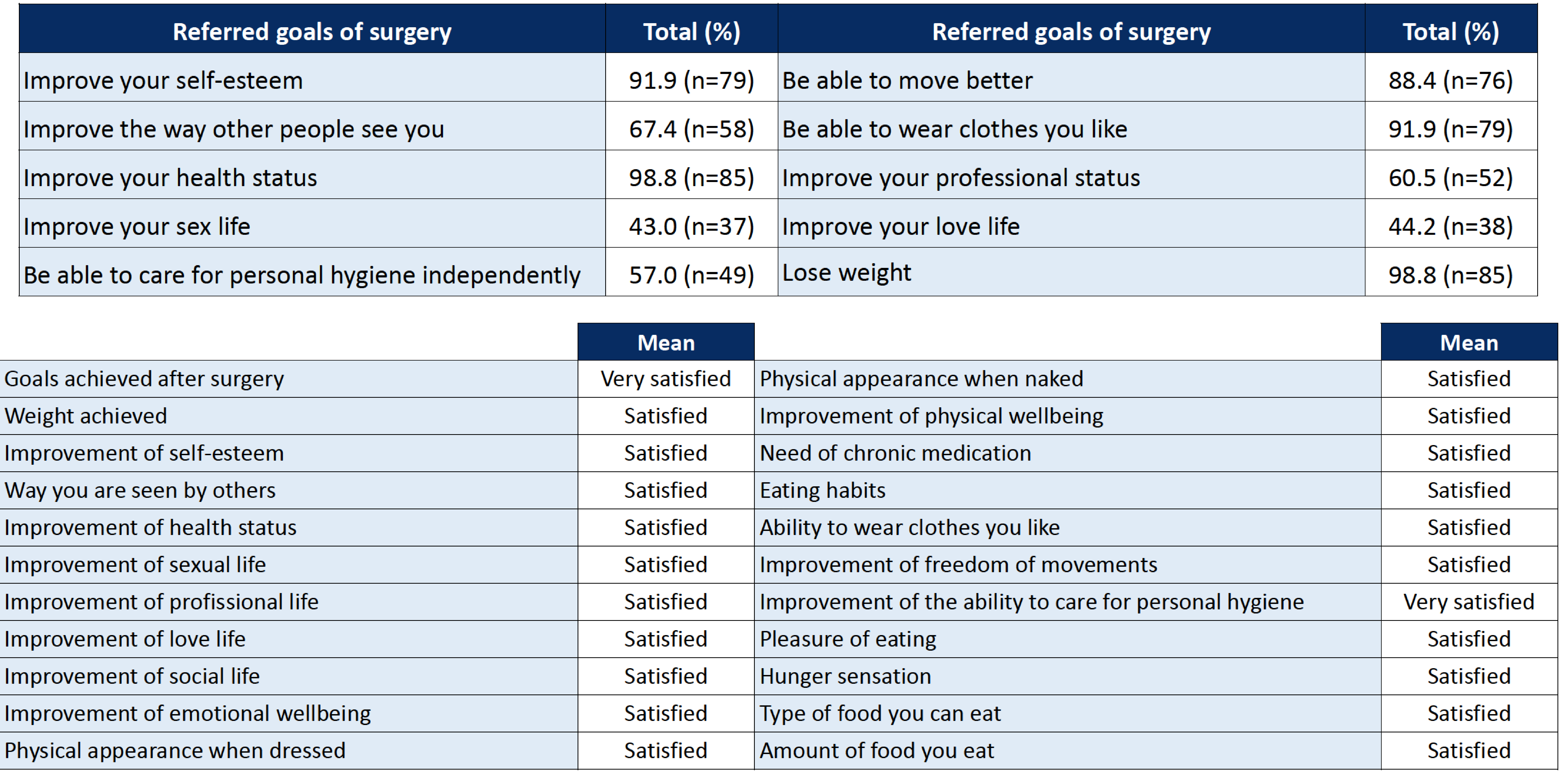

$34.9 \%$ of patients feel dissatisfied to very dissatisfied

with their "physical appearance when naked"

$12.8 \%$ of patients feel dissatisfied to very dissatisfied

with the "need of chronic medication"
Would you undergo surgery again?

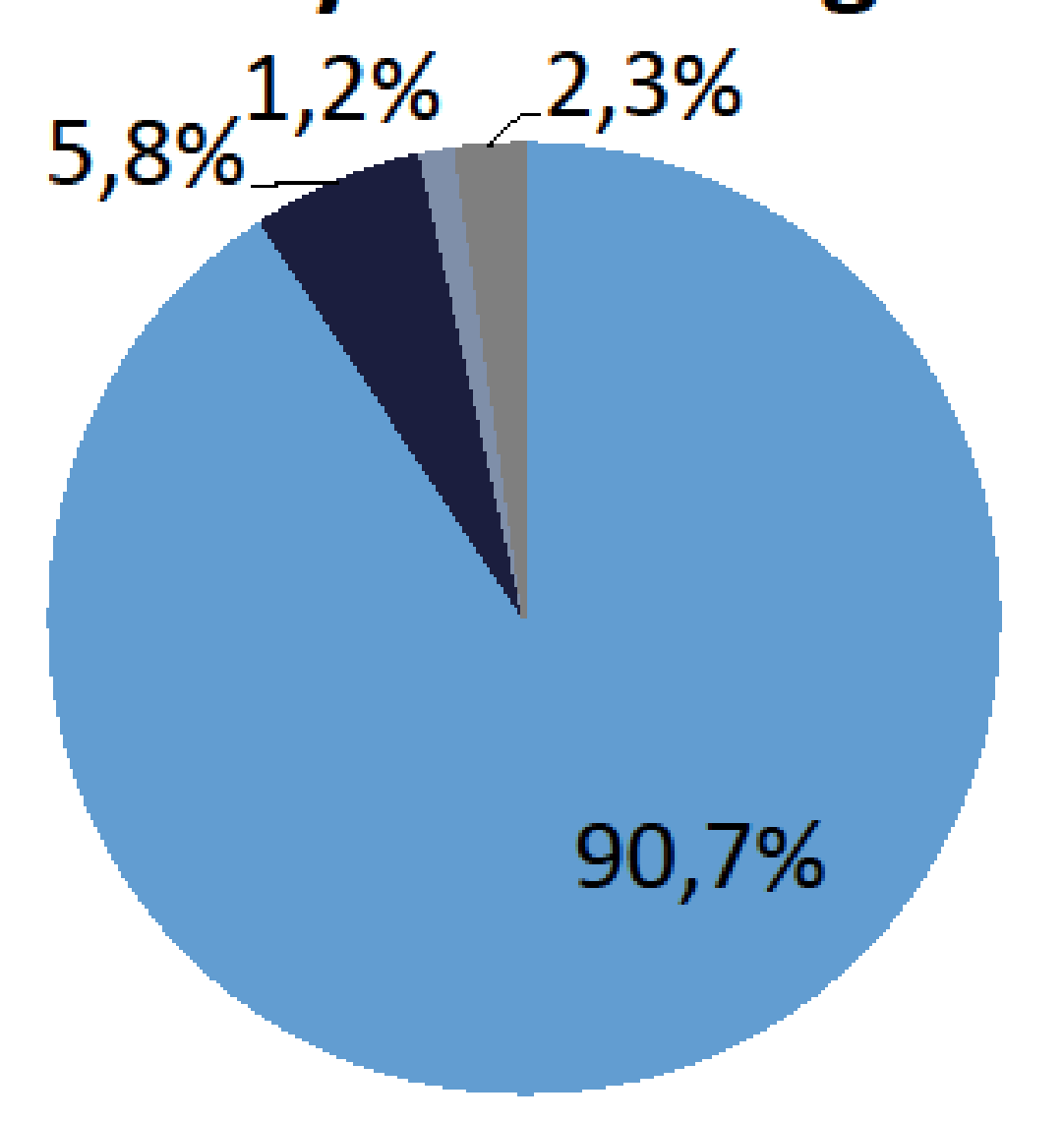

Would you recommend surgery to other patients?

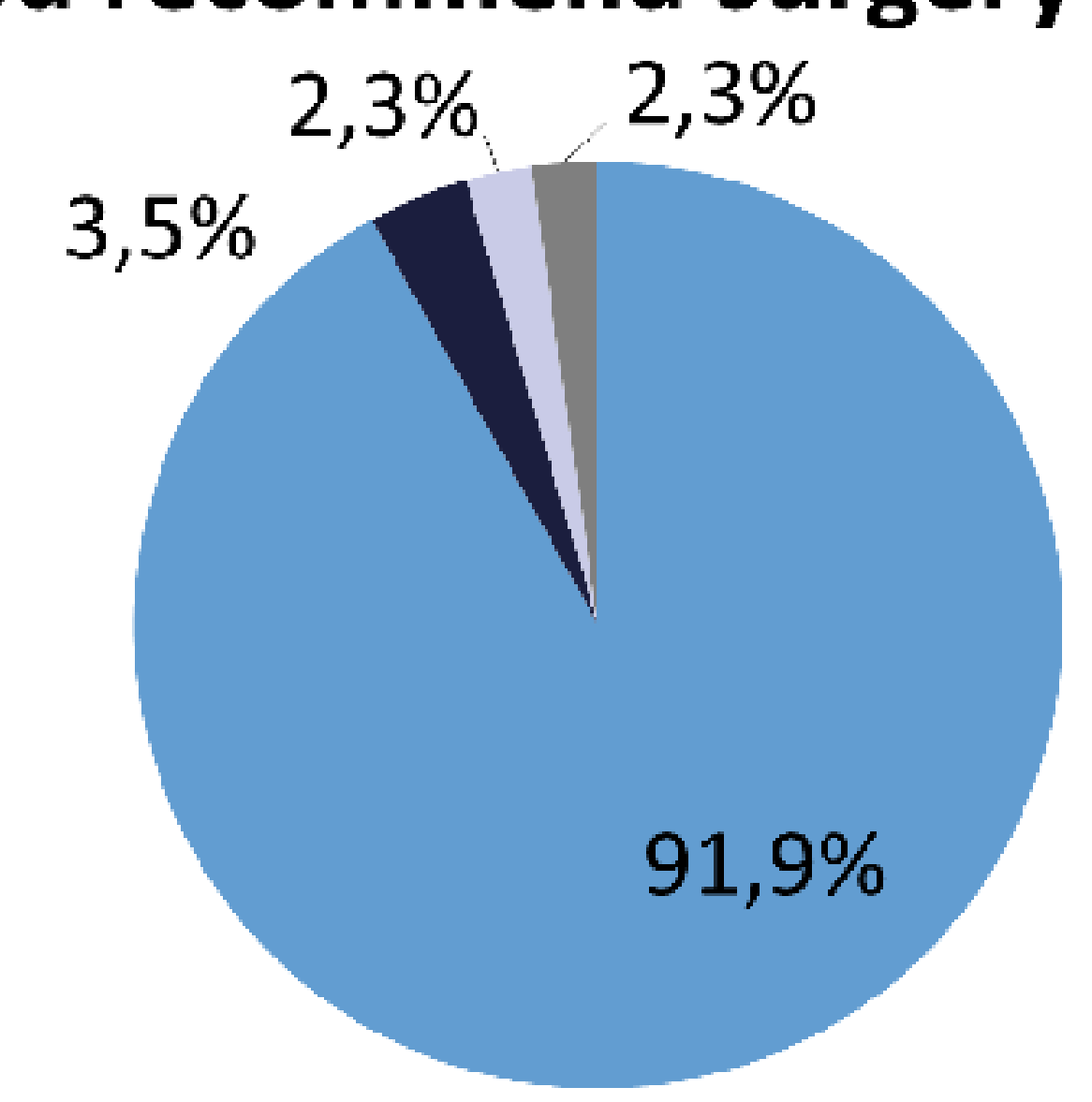

Yes, sure

- Yes, maybe

- I don't know

Maybe not

no, never

\section{CONCLUSION}

Patients were satisfied to very satisfied in all items tested, despite having unrealistic expectations. It is essential to assess what affects patients' satisfaction in order to understand their behavior and optimize our intervention. 